\title{
AN EMPTY CLASS OF NONMETRIC SPACES
}

\author{
ALAN DOW \\ (Communicated by Dennis Burke)
}

\begin{abstract}
Let CSSM be the class of compact nonmetrizable spaces in which every subspace of cardinality at most $\omega_{1}$ is metrizable. We show that CSSM is empty.
\end{abstract}

For the purposes of this article only let us call a space an SSM space (small subspaces metrizable) if it is not metrizable but it is regular and all of its subspaces of cardinality at most $\omega_{1}$ are metrizable. A CSSM space is a compact SSM space. If $X$ is a CSSM space, then $X$ is first countable $[\mathbf{H J}]$. Therefore under the continuum hypothesis $(\mathrm{CH})$ there are no CSSM spaces because, of course, a compact first countable space has cardinality at most $c$. This was first observed by Juhasz who then asked if the $\mathrm{CH}$ assumption could be removed [J]. It was shown in [D] that it is consistent with (and independent of) $\neg \mathrm{CH}$ that there are no Lindelö, countably compact or even $\omega_{1}$-compact first countable SSM spaces. In this article we show that there simply are never any CSSM spaces.

There is however an easy example, under $\mathrm{MA}+7 \mathrm{CH}$, of a Lindelöf first countable SSM space. I do not know if a Lindelof SSM space is necessarily first countable.

EXAMPLE 1. Recall that the Alexandroff double topology on $I \times 2$ (where $I$ is the unit interval) is obtained by declaring $I \times\{1\}$ to be open and discrete while a basic open neighbourhood of a point $(r, 0)$ is $U \times 2-\{(r, 1)\}$ where $r \in U$ is open in $I$. If $A \subset I$ is any uncountable set containing no uncountable closed set, then $X=(I-A \times\{0\}) \cup(A \times\{1\})$ is a Lindelof non metrizable subspace of the Alexandroff double.

Furthermore, if $\operatorname{MA}\left(\omega_{1}\right)$ is assumed then $X$ is an SSM space since $A \times\{1\}$ will be an $F_{\sigma}$-set in any subspace of $X$ of cardinality $\omega_{1}$ (see $[\mathbf{M}]$ ).

One might hope to modify the Alexandroff double somehow to obtain a CSSM space. In fact if $X$ were a CSSM space then $X$ would contain an uncountable discrete subset $D$; hence $\operatorname{cl} D$ would itself be a CSSM space. (To see that $X$ would contain such a $D$ see $2($ ii).)

2. PROPOSITION. If $X$ is a SSM space then:

(i) each separable subspace is metrizable and

(ii) $X$ contains an uncountable discrete subset and

(iii) if $X$ is, in addition, compact then $X$ contains an uncountable discrete set $D$ whose closure is a CSSM space.

Received by the editors June 3, 1987 and, in revised form, October 5, 1987.

1980 Mathematics Subject Classification (1985 Revision). Primary 54E35, 54E45.

Key words and phrases. Compact, metrizable, reflection.

This research was supported by N.S.E.R.C. Grant No. U0310. 
ProOF. (i) is essentially due to Hajnal and Juhasz [HJ]. They prove that a space has countable weight if all of its subspaces of cardinality at most $\omega_{1}$ do. Now (i) follows since if $K \subset X$ is separable, each subspace of size $\omega_{1}$ is contained in a separable metrizable space (since $X$ is SSM and $K$ is separable).

Of course (ii) is obvious since, by (i), $X$ is not separable and therefore $X$ has a non separable subspace of cardinality $\omega_{1}$. This subspace is metrizable, hence not ccc. For (iii), let $D$ be given by (ii) and note that $\operatorname{cl} D$ is not metrizable and is therefore a CSSM space.

3. CSSM is empty. Suppose that $X$ is a compact space containing the discrete space $\omega_{1}$ as a dense subspace. For each $x \in X$ fix a countable neighbourhood base $\{U(x, n): n<\omega\}$. Let $\mathscr{U}=\{\{U(x, n): n<\omega\}: x \in X\}$.

For each $\lambda<\omega_{1}$ let $\mathscr{C} \lambda=\bigcap\{\operatorname{cl}(\lambda-\alpha): \alpha<\lambda\}$.

We shall define by induction on $\gamma<\omega_{1}$ a continuous increasing sequence $\{M(\gamma)$ : $\left.\gamma<\omega_{1}\right\}$ of countable elementary submodels of some sufficiently large $H(\theta)$ and for each $\lambda(\gamma)=M(\gamma) \cap \omega_{1}$ we will choose $x(\gamma)$ in $\mathscr{C} \lambda(\gamma)$ as follows.

Suppose $\gamma<\omega_{1}$ and $\{M(\rho): \rho<\gamma\},\{x(\rho): \rho<\gamma\}$ have been chosen so that $\{X, \mathscr{U}\} \in M(\rho)$ and $\{x(\rho), M(\rho)\} \in M(\rho+1)$ for each $\rho<\gamma$.

In case $\gamma$ is a limit, let $M(\gamma)=\bigcup\{M(\rho): \rho<\gamma\}$. If $\gamma=\rho+1$ let $M(\gamma)$ be any countable elementary submodel of $H(\theta)$ containing $\{M(\rho), x(\rho)\}$.

The following fact is probably of some interest by itself and it provides the basis for the whole proof.

FACT 1. If $M$ is a countable elementary submodel of $H(\theta)$ such that $X, \mathscr{U}$ are in $M, \lambda=M \cap \omega_{1}$, and if $F \in[X \cap M]^{<\omega}$ and $p: F \rightarrow \omega$ are such that $\mathscr{C} \lambda \subset \bigcup\{U(x, p(x)): x \in F\}$ then

$$
\text { for some } \beta<\lambda \quad\left[\beta, \omega_{1}\right) \subset \bigcup\{U(x, p(x)): x \in F\} \text {. }
$$

PROOF. By definition of $\mathscr{C} \lambda$, each sequence cofinal in $\lambda$ is eventually in $\bigcup\{U(x, p(x)): x \in F\}$, hence there is some $\beta<\lambda$ such that $[\beta, \lambda) \subset \bigcup\{U(x, p(x)): x \in$ $F\}$. But since $F, p$ and $\omega_{1}$ are all in $M$, we have that $M$ is a model of $\left[\beta, \omega_{1}\right) \subset$ $\bigcup\{U(x, p(x)): x \in F\}$. Now the fact follows since $M$ is an elementary submodel.

NOTATION. For $\rho<\gamma$ let $U(\rho, n)=U(x(\rho), n)$.

FACT 2. There is an $x(\gamma) \in \mathscr{C} \lambda(\gamma)$ such that for any $\rho<\gamma$ and $n<\omega$, $x(\gamma) \in U(\rho, n) \rightarrow \lambda(\gamma) \in U(\rho, n)$.

PROOF. If not we could find for each $x \in \mathscr{C} \lambda(\gamma)$ a pair $t(x) \in \gamma \times \omega$ such that $x \in U(t(x))$ and $\lambda(\gamma) \notin U(t(x))$. Since $\mathscr{C} \lambda(\gamma)$ is compact we find $F \in[\gamma]^{<\omega}$ such that $\bigcup\{U(t(x)): x \in F\} \supset \mathscr{C} \lambda(\gamma)$. However, this contradicts Fact 1 since $\lambda(\gamma) \notin U(t(x))$ for $x \in F$.

Therefore we have defined a cub $\left\{\lambda(\gamma): \gamma<\omega_{1}\right\}$, a sequence $\left\{x(\gamma): \gamma<\omega_{1}\right\}$ with $x(\gamma) \in \mathscr{C} \lambda(\gamma)$ and a sequence of neighbourhood bases $\left\{U(\gamma, n): \gamma<\omega_{1}, n<\omega\right\}$ so that $\rho<\gamma$ and $x(\gamma) \in U(\rho, n) \rightarrow \lambda(\gamma) \in U(\rho, n)$.

FACT 3. $\omega_{1} \cup\left\{x(\gamma): \gamma<\omega_{1}\right\}$ is not metrizable.

PrOOF. Assume that it is metrizable. Recall that each open subset of a metric space is an $F_{\sigma}$. Therefore there must be a stationary set $S \subset\left\{\lambda(\gamma): \gamma<\omega_{1}\right\}$ such that $\operatorname{cl} S \cap\left\{x(\gamma): \gamma<\omega_{1}\right\}=\varnothing$ since $\omega_{1}$ is open. For each $\lambda(\gamma) \in S$ choose $n(\gamma)<\omega$ so that $U(x(\gamma), n(\gamma)) \cap S=\varnothing$. It follows that if $\lambda(\gamma)<\lambda(\rho)$ are both in $S$ then $x(\rho) \notin U(x(\gamma), n(\gamma))$ since $\lambda(\rho) \notin U(x(\gamma), n(\gamma))$. Let, for $n \in \omega, \mathscr{V}_{n}$ be a locally finite family of open subsets of $\omega_{1} \cup\left\{x(\gamma): \gamma<\omega_{1}\right\}$ such that $\bigcup\left\{\mathscr{V}_{n}: n \in \omega\right\}$ is a 
base (recall that each metric space has a $\sigma$-locally finite base). For each $\lambda(\gamma) \in S$, there is an $m_{\gamma}$ and a $V_{\gamma} \in \mathscr{V}_{m_{\gamma}}$ such that $x(\gamma) \in V_{\gamma} \subset U(x(\gamma), n(\gamma))$. There is an $m \in \omega$ such that $S^{\prime}=\left\{\lambda(\gamma) \in S: m_{\gamma}=m\right\}$ is stationary. Since $\lambda(\gamma)<\lambda(\rho)$ both in $S^{\prime}$ implies $x(\rho) \notin V_{\gamma}$, these sets are all distinct (i.e. $V_{\gamma} \neq V_{\rho}$ ). However, for each $\lambda(\gamma) \in S^{\prime}, V_{\gamma} \cap[0, \lambda(\gamma)) \neq \varnothing$ since $x(\gamma)$ is a limit point of $[0, \lambda(\gamma))$. Now a pressing down argument gives that the family $\left\{V_{\gamma}: \lambda(\gamma) \in S^{\prime}\right\}$ is not point-finite, contradicting that $\mathscr{V}_{m}$ is locally finite.

FACT $4 . X$ is not SSM.

\section{REFERENCES}

[D] A. Dow, Two applications of reflection and forcing to topology, General Topology and its Relations to Modern Analysis and Algebra VI; Proc. Sixth Prague Topological Sympos. 1986, ed. Z. Frolik, Heldermann-Verlag, Berlin, 1988.

[HJ] I. Juhasz, Cardinal functions in topology -10 years later, Mathematisch Centrum, Amsterdam, 1980.

[J] _ Cardinal functions II, Handbook of Set Theoretic Topology, ed. K. Kunen and J. E.Vaughan, North-Holland, Amsterdam, 1984, pp. 63-110.

[M] A. Miller, Special subsets of the real line, Handbook of Set Theoretic Topology, ed. K. Kunen and J. E. Vaughan, North-Holland, Amsterdam, 1984, pp. 201-234.

Department of Mathematics, YORK University, TORONTO, CANAdA, M3J 1P3 